\title{
IMPACTO DA SAÚDE BUCAL NA QUALIDADE DE VIDA DE ADOLESCENTES
}

\author{
IMPACT OF ORAL HEALTH QUALITY OF LIFE ADOLESCENT
}

\author{
Mônica Ciler Gomes Pereira, Fábio Silva de Carvalho, Cristiane Alves Paz de Carvalho
}

Universidade Estadual do Sudoeste da Bahia - UESB

\section{Abstract}

The aim of this study was to evaluate how the oral health of adolescent influences the quality of their lives. A descriptive cross-sectional study was realized, with a sample of adolescent students aged between 12 and 15 years, from the city of Ibirataia and district of Cotton on the state Bahia, Brazil, who completed a validated questionnaire, the Oral Health Impact Profile (OHIP-14). It was observed a general average of 4,97 for the total score of the OHIP-14. The areas with the highest and lowest average were physical pain $(1,16)$ and functional limitation (0,43), respectively. Most respondents reported some impact of oral conditions on quality of life $(90,00 \%)$, however, most of these reported low impact (74,28\%). Other 15,70\% reported a average impact and 9,99\% no impact. There were not reports of major impact.The oral health impacted the quality of life of most teenagers, although this impact has been weak. This suggests that, even if present in the daily activities of adolescents participating in this study, it does not cause major effects on the quality their lives. The physical pain and psychological distress were the most prevalent areas.

\section{Resumo}

Objetivou-se avaliar como a saúde bucal de adolescentes escolares influencia na qualidade de vida destes, por meio das seguintes dimensões: limitação funcional, dor física, desconforto psicológico, incapacidade psicológica, incapacidade social e desvantagem social. Realizou-se um estudo descritivo do tipo transversal, sendo a amostra composta por adolescentes escolares com idade entre 12 e 15 anos, do município de Ibirataia-BA e do distrito de Algodão-BA, os quais responderam a um questionário validado, o Oral Health Impact Profile (OHIP-14). Observou-se média geral de 4,97 para o escore total do OHIP-14. Os domínios que apresentaram maior e menor média foram dor física $(1,16)$ e limitação funcional $(0,43)$, respectivamente. A maioria dos entrevistados relatou algum impacto das condições bucais na qualidade de vida $(90,00 \%)$, entretanto, a maior parte destes relatou fraco impacto (74,28\%). Outros 15,70\% relataram médio impacto e 9,99\% sem impacto. Não houve relato de impacto forte. Embora o impacto das condições bucais esteja presente nas atividades diárias dos adolescentes participantes deste estudo, este não causa grandes efeitos na qualidade de vida dos mesmos. A saúde bucal causou impacto na qualidade de vida da maioria dos adolescentes, embora este impacto tenha sido fraco. A dor física e o desconforto psicológico foram os domínios mais prevalentes.

Palavras chave: Adolescência; Qualidade de vida; Saúde Bucal. 


\section{Introdução}

A saúde geral abrange a saúde bucal, sendo esta considerada fundamental para a qualidade de vida. A condição adequada de saúde bucal é importante para realização de atividades como falar, mastigar, reconhecer o sabor dos alimentos, sorrir, viver livre de dor e desconforto, e se relacionar com outras pessoas sem constrangimento ${ }^{1}$

Normalmente, os indivíduos só compreendem a relevância da saúde bucal para a qualidade de vida diante de perspectivas que envolvam os domínios físico, social e psicológico. Ocorrência de dor e desconforto e capacidade de se alimentar são consideradas, respectivamente, os aspectos negativos e positivos mais relevantes para a qualidade de vida ${ }^{2}$.

O conceito de qualidade de vida no âmbito da saúde, visto no seu sentido mais amplo, finca suas bases na compreensão das necessidades humanas materiais, fundamentais e espirituais, sendo o conceito de promoção da saúde seu foco mais relevante ${ }^{3}$. A Organização Mundial de Saúde (OMS) conceitua qualidade de vida como a percepção do indivíduo sobre sua posição na vida, nos sistemas de valores nos quais ele vive, no contexto da cultura e em relação a seus objetivos, padrões, preocupações e expectativas. Além de compreender uma representação subjetiva da sensação de bem-estar, o conceito de qualidade de vida é multidimensional e inclui dimensões positivas e negativas ${ }^{4,5}$.

Na maioria das vezes, as condições de saúde bucal são analisadas em modelos biomédicos, baseados estritamente em parâmetros clínicos, os quais são de grande eficácia na avaliação das patologias, porém limitados em designar o impacto da doença e como esta pode refletir na qualidade de vida destes indivíduos. Tendo em vista o conceito ampliado em saúde e como esta deve ser interpretada de modo multidimensional, é necessário considerar toda a complexidade biológica, psicológica e social ${ }^{6}$.

A crescente necessidade de determinar a repercussão integral de alterações bucais levou ao desenvolvimento dos instrumentos de avaliação da qualidade de vida relacionada à saúde bucal, os quais estão conquistando espaço cada vez maior em pesquisas odontológicas. Contudo, boa parte dos índices desenvolvidos era voltada, em sua maioria, para pacientes adultos ou idosos, sendo que a qualidade de vida relacionada à saúde bucal em adolescentes e crianças permaneceu por mais tempo desconhecida, tornando-se objeto de estudo de instrumentos desenvolvidos mais recentemente ${ }^{5}$.

A adolescência pode ser considerada um período de mudança que influencia de forma significativa os aspectos físicos, sexuais, cognitivos e emocionais ${ }^{7}$ É possível compreender a adolescência como um período do desenvolvimento situado entre a infância e a fase adulta, delimitado pela OMS como a faixa etária dos 10 aos 19 anos de idade ${ }^{8}$. É uma fase de reorganização emocional, em que os conflitos resultantes de alterações biológicas, psíquicas e sociais são comumente exacerbados através do comportamento ${ }^{7}$.

Toda essa complexidade pode ser constatada pelo cirurgião-dentista por meio de manifestações de negligência com a higiene bucal, irreverência frente às recomendações para uma dieta mais equilibrada e menos cariogênica, não comparecimento às consultas e relevante falta de interesse pelo atendimento odontológico, exceto quando este envolve uma proposta de melhora da estética ${ }^{9}$. Para lidar com essa faixa etária, o cirurgião-dentista necessita de técnica, conhecimento e, sobretudo, sensibilidade para compreender o contexto no qual o adolescente encontra-se inserido e como este pode interferir nas suas condições de saúde bucal.

Atualmente, há uma diversidade de instrumentos de mensuração da qualidade de vida relacionada à saúde bucal, como o DIDL (Dental Impactson Daily Living), o OHIP (The Oral Health Impact Profile) e a sua versão abreviada o OHIP-14 e o OIDP (Oral Impactson Daily Performances), sendo que grande parte desses questionários foram elaborados para aplicação em adultos, porém, o OHIP e o OIDP foram empregados em pesquisas que contemplaram adolescentes $^{10}$.

O OHIP é um dos instrumentos que avalia a percepção dos indivíduos com relação ao impacto negativo consequente das manifestações bucais no seu bem-estar. As informações do OHIP buscam acrescentar os indicadores tradicionais de epidemiologia bucal e proporcionar um perfil mais detalhado do impacto da doença nas populações. Apresenta em sua estrutura sete dimensões e 49 itens, sendo um dos instrumentos mais utilizados na área da Odontologia, por ser alicerçado em uma base conceitual e empírica, ter propriedades psicométricas conhecidas e ser de fácil aplicação. Foi utilizado em pesquisas em países como Canadá e Estados Unidos, tendo posteriormente sua versão reduzida para 14 itens - OHIP-14 ${ }^{11}$.

A construção de estudos na área de 
qualidade de vida poderá subsidiar mudanças nas práticas assistenciais e no embasamento de novos modelos do processo saúde-doença. A análise da qualidade de vida pode aperfeiçoar a relação profissional-paciente a partir de uma assimilação mais ampla do processo de doença, localizando as necessidades e melhorando o discernimento, por parte do profissional, e a confiança pelo paciente ${ }^{9}$.

Considerando-se a relevância de verificar como a saúde bucal pode influenciar na qualidade de vida do adolescente por meio de uma perspectiva multidimensional, e ao mesmo tempo proporcionar aos profissionais o planejamento e a prática de programas mais resolutivos de prevenção e promoção em saúde bucal para adolescentes, justifica-se a importância do presente estudo. Desta forma, objetivou-se avaliar como a saúde bucal de adolescentes escolares do município de Ibirataia$\mathrm{BA}$ e do distrito de Algodão-BA pode influenciar na qualidade de vida destes.

\section{Metodologia}

A coleta de dados deste estudo foi realizada somente após aprovação do projeto pelo Comitê de Ética em Pesquisa da Universidade Estadual do Sudoeste da Bahia - CEP/UESB (parecer 513.574 CAAE 26743214.7.0000.0055) e após autorizações concedidas pelos pais/responsáveis dos participantes, mediante o preenchimento do Termo de Consentimento Livre e Esclarecido (TCLE). Além disso, foi aplicado o Termo de Assentimento para menores de 18 anos, a fim de obter o consentimento dos mesmos para cooperação com a pesquisa.

Esta pesquisa caracterizou-se como um estudo descritivo do tipo transversal, realizado com adolescentes regularmente matriculados em escolas municipais do município de Ibirataia-BA e distrito de Algodão-BA. Segundo informações da Secretaria Municipal de Educação, o município e o distrito dispõem de 10 escolas municipais e haviam 1.847 adolescentes matriculados, na faixa etária de 12 a 19 anos de idade, para o primeiro semestre letivo de 2014. Este estudo foi desenvolvido com adolescentes na faixa etária de 12 a 15 anos de idade e para se estimar o número de adolescentes necessários para a composição de uma amostra representativa do município e distrito, foi feito o cálculo da amostra. Após o cálculo, obteve-se uma amostra representativa de 142 adolescentes. dentre as 10, uma única escola localiza-se no distrito de Algodão-BA, sendo, portanto, automaticamente selecionada. Como o município de Ibirataia-BA apresenta 9 escolas municipais, foi realizado um sorteio para seleção de 2 escolas, adotando como critério a localização: uma das escolas deveria localizar-se na periferia e outra na região central do município.

A escolha da técnica de coleta de dados foi adequada ao objeto de estudo e, portanto, nesta pesquisa, utilizou-se um questionário. O questionário escolhido para avaliar o impacto da saúde bucal na qualidade de vida dos adolescentes foi o Oral Health Impact Profile em sua versão reduzida (OHIP-14) e validada para o português. O OHIP-14 consiste em um dos instrumentos de avaliação da percepção das pessoas sobre o impacto negativo decorrente dos acometimentos bucais no seu bem-estar.

O questionário foi entregue por meio de abordagem direta, ou seja, a pesquisadora entregou pessoalmente o documento, que foi respondido pelo próprio participante, sem qualquer interferência, permitindo que ele se expressasse livremente.

As dimensões do impacto da qualidade vida avaliadas por este questionário são: limitação funcional, dor física, desconforto psicológico, incapacidade física, incapacidade psicológica, incapacidade social e deficiência. Para cada resposta foi atribuído um peso, conforme Slade ${ }^{11}$.

Para análise, os dados foram processados em planilha do programa Office Excel $2010^{\circ}$. Quanto à mensuração do impacto da saúde bucal na qualidade de vida, foram conferidas pontuações originais do OHIP-14 para cada pergunta de acordo com a resposta apresentada: Nunca - 0; Raramente - 1; Às vezes - 2; Quase sempre - 3; Sempre - 4. As respostas foram multiplicadas pelo respectivo peso de cada questão e em seguida somadas, produzindo um escore total do OHIP-14, que pode variar de 0 a 28 , sendo que, quanto maior a pontuação, maior a percepção do impacto pelo indivíduo ${ }^{12}$. Assim, o valor máximo alcançado pra cada dimensão é 4. Além disso, utilizou-se uma escala de impacto das condições de saúde bucal na qualidade de vida, proposta por Bastos ${ }^{13}$, cujo impacto para cada dimensão foi classificado em: sem impacto (0), fraco $(0<$ fraco $\leq 9)$, médio $(9<$ médio $\leq 18)$ e forte $(18<$ forte $\leq 28)$.

Os resultados foram apresentados de acordo com análise descritiva, baseada em proporções e frequências absolutas e relativas.

Quanto à seleção das escolas municipais, 


\section{Resultados e Discussões}

Foram distribuídos 300 termos de consentimento para todas as turmas, nos turnos matutino e vespertino, que contemplavam adolescentes com faixa etária entre 12 e 15 anos, nas três escolas participantes. Deste total, 70 termos retornaram devidamente assinados pelos pais/responsáveis, sendo a taxa de resposta de $23,33 \%$. O principal motivo das perdas foi o não retorno dos termos de consentimento corretamente preenchidos e falta de interesse dos pais em autorizar a participação dos filhos.

A composição final da amostra (Tabela 1) deste estudo ( $\mathrm{n}$ ) foi de 70 adolescentes, sendo a faixa etária mais prevalente a de 12 anos (40,00\%). Quanto ao gênero, a maioria dos participantes era meninas (61,42\%). A maior parte dos adolescentes relatou residir no distrito de Algodão-BA (81,42\%) e na zona urbana (81,42\%).

\begin{tabular}{lcc}
\hline \multicolumn{3}{c}{ Tabela 1 - Caracterização da amostra de acordo com a idade, gênero, cidade e m } \\
\cline { 2 - 3 } & $\mathbf{n}$ & \% \\
\hline Idade & 28 & 40,00 \\
12 & 21 & 30,00 \\
13 & 14 & 20,00 \\
14 & 7 & 10,00 \\
15 & & \\
Gênero & 43 & 61,42 \\
Feminino & 27 & 38,57 \\
Masculino & & \\
Cidade & 13 & 18,57 \\
Ibirataia & 57 & 81,42 \\
Algodão & & \\
Moradia & & 81,42 \\
Urbana & 57 & 18,57 \\
Rural & 13 & $\mathbf{1 0 0 , 0 0}$ \\
\hline Total & $\mathbf{7 0}$ &
\end{tabular}

Ao avaliar o impacto da saúde bucal na qualidade de vida dos adolescentes, observou-se média geral de 4,97, sendo as pontuações mínima e máxima obtidas no escore total dos questionários analisados, 0,00 e 16,49, respectivamente. 0 domínio que apresentou maior média foi dor física $(1,16)$, tanto para a amostra geral quanto para as diferentes idades, gênero, cidade e moradia, sendo, portanto, a que mais contribuiu para a média final do OHIP-14. Já o domínio que apresentou menor média foi limitação funcional $(0,43)$, conforme pode ser observado na Tabela 2.

Tabela 2 - Distribuição da média das dimensões do OHIP-14.

\begin{tabular}{|c|c|c|c|c|c|c|c|c|}
\hline & LF & DF & DP & IF & IP & IS & D & $\mathbf{T}$ \\
\hline Idade & Média & Média & Média & Média & Média & Média & Média & Média \\
\hline 12 & 0,39 & 1,21 & 0,98 & 0,52 & 0,66 & 0,47 & 0,59 & 4,82 \\
\hline 13 & 0,53 & 1,15 & 1,00 & 0,55 & 0,61 & 0,62 & 0,62 & 5,07 \\
\hline 14 & 0,22 & 1,07 & 1,30 & 0,79 & 0,61 & 0,30 & 0,47 & 4,77 \\
\hline 15 & 0,71 & 1,14 & 1,21 & 0,94 & 0,57 & 0,41 & 0,71 & 5,70 \\
\hline \multicolumn{9}{|l|}{ Gênero } \\
\hline Feminino & 0,51 & 1,25 & 1,29 & 0,76 & 0,77 & 0,43 & 0,59 & 5,59 \\
\hline Masculino & 0,30 & 1,00 & 0,73 & 0,42 & 0,40 & 0,55 & 0,59 & 3,99 \\
\hline \multicolumn{9}{|l|}{ Cidade } \\
\hline Ibirataia & 0,46 & 1,20 & 1,03 & 0,97 & 0,69 & 0,69 & 0,57 & 5,61 \\
\hline Algodão & 0,42 & 1,14 & 1,08 & 0,55 & 0,61 & 0,43 & 0,59 & 4,83 \\
\hline \multicolumn{9}{|l|}{ Moradia } \\
\hline Urbana & 0,43 & 1,19 & 1,08 & 0,60 & 0,69 & 0,50 & 0,56 & 5,05 \\
\hline Rural & 0,42 & 1,00 & 1,04 & 0,73 & 0,35 & 0,38 & 0,72 & 4,65 \\
\hline Total & 0,43 & 1,16 & 1,07 & 0,63 & 0,63 & 0,47 & 0,59 & 4,97 \\
\hline
\end{tabular}

LF (limitação funcional), DF (dor física), DP (desconforto psicológico), IF (incapacidade física), IP (incapacidade psicológica), IS (incapacidade social), D (deficiência), T (total). 
Quanto ao impacto da saúde bucal na qualidade de vida, a prevalência dos entrevistados que relataram algum impacto das condições bucais na qualidade de vida foi de $90,00 \%$. Sendo que, a maioria, correspondentes a adolescentes com faixa etária de 12 anos, gênero feminino, residentes em Algodão e na zona urbana, relatou fraco impacto $(74,28 \%)$. Outros $15,70 \%$ relataram médio impacto, apresentando estes, 13 anos de idade, gênero feminino, residentes na cidade de Algodão e localidade urbana. Para 9,99\% dos adolescentes, a saúde bucal não causou impacto na qualidade de vida e não houve relato de impacto forte da saúde bucal na qualidade de vida no grupo de adolescentes participantes deste estudo (Tabela 3).

Tabela 3 - Impacto da saúde bucal na qualidade de vida de acordo com a idade, gênero, cidade e moradia.

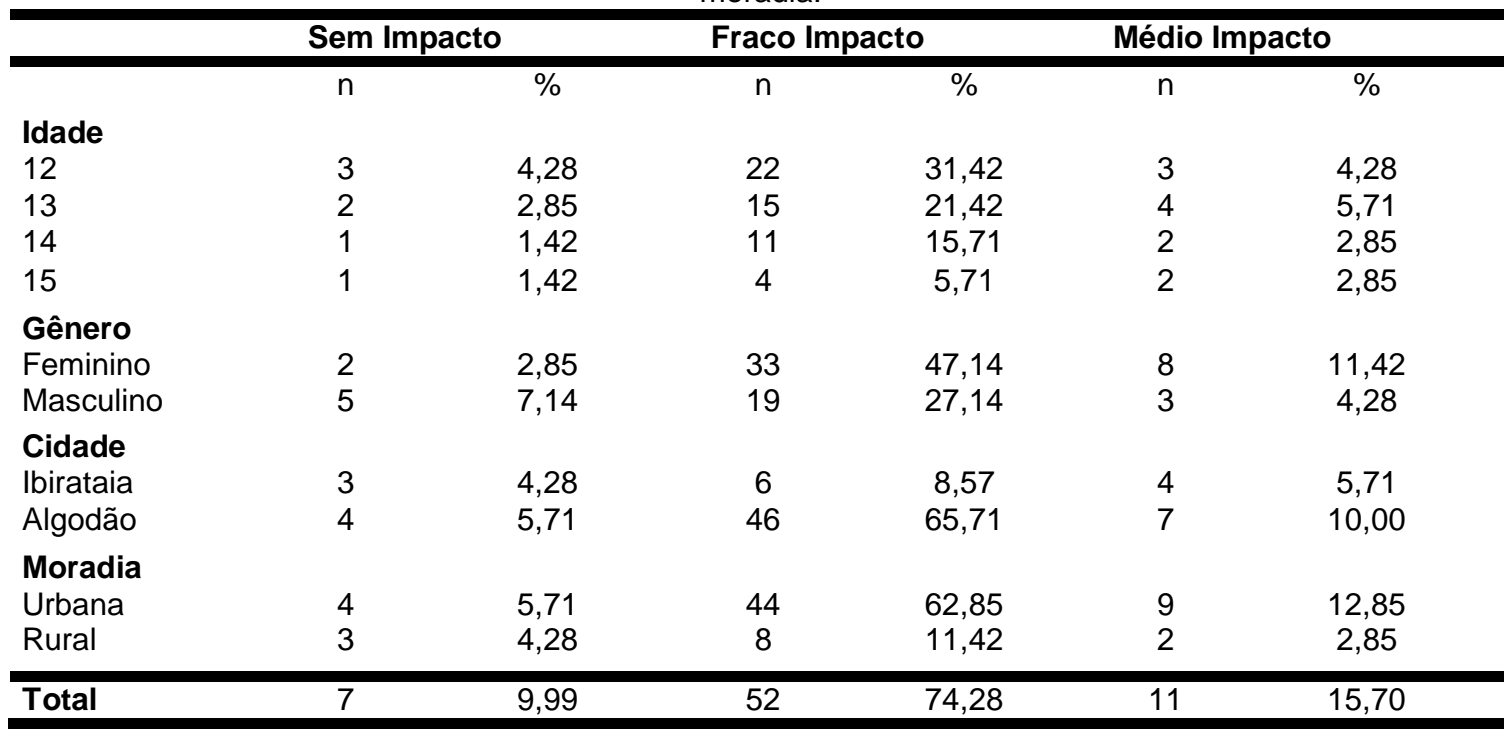

A saúde bucal dos adolescentes tem sido avaliada a partir de investigações que levam em consideração condições meramente objetivas da mesma. Tais investigações são direcionadas para compreensão das condições dentárias e/ou bucais e na avaliação de tratamentos realizados, porém, limitadas em designar o quanto as pessoas se sentem afetadas pela sua condição bucal. A partir desse pressuposto, que reconhece a limitação de estudos focados exclusivamente em parâmetros clínicos, pesquisas que investigam os problemas de saúde bucal como importantes causadores de impacto negativo no desempenho de atividades diárias e na qualidade de vida dos indivíduos, têm conquistado significativo espaço nos estudos epidemiológicos ${ }^{1}$.

Com base na necessidade de zelar mais das pessoas, buscou-se nos indicadores subjetivos em saúde bucal, uma alternativa para melhor compreender as carências, uma vez que, conseguem capturar as dificuldades relatadas pelos indivíduos. O OHIP-14 caracteriza-se por refletir de forma eficiente as experiências subjetivas associadas à saúde bucal, a percepção da experiência de saúde e doença e outros sintomas como problemas na vida das pessoas, sendo, atualmente, um dos instrumentos mais utilizados para mensurar o impacto adverso provocado por condições bucais no bem-estar e na qualidade de vida ${ }^{14}$.

No presente estudo, notou-se que $90,00 \%$ dos entrevistados relataram algum impacto da saúde bucal na qualidade de vida. Krisdaponget al. ${ }^{15}$, em estudo conduzido com adolescentes tailandeses com faixa etária de 15 anos, utilizando o OIDP como instrumento avaliador, encontraram que $83,00 \%$ dos entrevistados relataram ter algum impacto da saúde bucal na qualidade de vida, nos últimos seis meses.

A maioria dos adolescentes entrevistados neste estudo $(74,28 \%)$ relatou fraco impacto, sendo estes, correspondentes à faixa etária de 12 anos, gênero feminino, residentes em Algodão e na zona urbana. Este resultado foi inferior ao estudo realizado por Bastos et al. (2012), em que $87,95 \%$ dos adolescentes de 15 a 19 anos de Bauru, São Paulo (SP), relataram fraco impacto da saúde bucal na qualidade de vida. No entanto, resultados semelhantes foram encontrados em estudo proposto por Monteiro ${ }^{16}$, em que $74,22 \%$ dos adolescentes, entre 15 e 19 anos, regularmente matriculados em escolas estaduais do município de Agudos (SP), apresentaram fraco 
impacto das condições de saúde bucal na qualidade de vida.

O baixo escore médio encontrado para o OHIP-14 neste estudo $(4,97)$ demonstra que o impacto da saúde bucal na qualidade de vida, embora presente, se mostrou de baixa intensidade, sem grande repercussão para esses adolescentes.

Os domínios que apresentaram maior média, tanto para a amostra geral quanto para as diferentes idades, gênero, cidade e moradia, foram dor física $(1,16)$ e desconforto psicológico $(1,07)$. Resultados semelhantes também foram verificados em estudo conduzido com adolescentes de 15 a 19 anos, residentes nas áreas urbana e rural do Município de Montes Claros, Minas Gerais, Brasil, no ano de $2008^{1}$.

Outro estudo que utilizou o OHIP-14 como instrumento para mensuração do impacto da saúde bucal na qualidade de vida, também apontou maior significância na dimensão "dor física" ${ }^{14}$. O domínio dor física revela o quanto alterações na saúde bucal causam dor no dente ou desconforto ao comer.

Estudo realizado com adolescentes de 15 a 19 anos, utilizando o OHIP-14 para mensuração da qualidade de vida e demais marcadores clínicos para avaliar as condições de saúde bucal, dentre eles o Índice CPO-D (Dentes permanentes cariados, perdidos e obturados) para cárie dentária, também demonstrou que os domínios desconforto psicológico e dor física foram os de maior influência das condições de saúde bucal na qualidade de vida; ambos os domínios apresentaram relação significativa com 0 componente perdido do CPO-D. Estes resultados podem sugerir que a perda dentária está relacionada com o impacto que a saúde bucal pode causar na presença de dor e uma preocupação evidente dos adolescentes com as futuras consequências de alterações nas condições de saúde bucal ${ }^{16}$.

Estas evidências mostraram uma limitação do presente estudo, e sinalizaram para a importância da utilização de indicadores clínicos, bem como da avaliação epidemiológica, do acesso aos serviços de saúde, da necessidade de tratamento, entre outras variáveis, que permitam associações com o impacto da saúde bucal na qualidade de vida. Desta forma, mais estudos fazem-se necessários.

O domínio limitação funcional foi o que apresentou menor média $(0,43)$. Este domínio refere-se ao quanto as alterações bucais podem estar afetando atividades como falar e sentir o sabor dos alimentos.

Estudo que analisou dados extraídos de formulários de exames de cárie dentária, além de questionários de necessidade percebida (OHIP14) de uma amostra de 312 escolares de 12 anos de idade da rede pública, demostrou que o OHIP apresenta um bom valor preditivo da declaração de necessidade de tratamento odontológico, indicando uma boa validade de conteúdo. A dimensão dor física foi a que apresentou melhor valor preditivo. A razão de chances mostrou que um indivíduo que declarou dor física no OHIP apresentou chance aproximadamente 1,5 vezes maior de declarar necessidade de tratamento comparado ao indivíduo que não declarou essa dimensão. Esse resultado indicou boa validade de conteúdo do OHIP frente ao indicador global da percepção declarada de necessidade de tratamento odontológico. Considerando-se a predição de acordo com o modelo logístico, a chance de uma pessoa que declarou dor física também declarar necessidade de tratamento odontológico foi alta, em relação ao indivíduo que não declarou essa dimensão ${ }^{17}$.

Estudo realizado com adolescentes, que buscou avaliar as más-oclusões e seu impacto na qualidade de vida, mostrou que a gravidade das más-oclusões impactou negativamente na qualidade de vida, mas em razão inferior à influência da condição estética. A estética ruim, autoavaliada e graduada pelo examinador, foi o preditor mais forte para o impacto negativo na qualidade de vida do que a gravidade das másoclusões e a busca pelo tratamento ortodôntico $^{18}$.

Dados do Levantamento Epidemiológico Nacional de Saúde Bucal-SB-Brasil 2010, utilizando o OIDP como instrumento de avaliação, apontou que, aos 12 anos de idade, $34,80 \%$ dos adolescentes apresentaram algum impacto da saúde bucal na qualidade de vida. Dificuldade para comer, incômodo para escovar os dentes, apresentar-se irritado ou nervoso e vergonha para sorrir foram os impactos mais prevalentes em todas as regiões e no país, com quadro semelhante entre 15 e 19 anos de idade $^{19}$.

Uma alternativa para a elevada prevalência de impactos dos problemas bucais associados à qualidade de vida está certamente relacionada ao acesso a tratamento odontológico abrangente, que inclui tratamento especializado para as pessoas com alta prevalência de necessidades clínicas e percebidas e, particularmente, para grupos com contexto socioeconômico desvantajoso ${ }^{14}$. 
Desta forma, é notória a importância de uma abordagem multidimensional na avaliação da saúde bucal de uma população. Além dos parâmetros objetivos e clínicos, é essencial levar em consideração as particularidades da população estudada, o meio no qual ela se insere, seus padrões e preocupações. Um número significativo de instrumentos válidos e confiáveis tem sido utilizado atualmente e são capazes de captar aspectos subjetivos da saúde bucal e seu impacto nas dimensões funcional, social e psicológica, dentre eles destaca-se o OHIP-14 ${ }^{1}$.

\section{Conclusões}

A saúde bucal causou impacto na qualidade de vida da maioria dos adolescentes, embora este impacto tenha sido fraco. Isto sugere que, mesmo presente nas atividades diárias dos adolescentes, o impacto das condições bucais não causa grandes efeitos na qualidade de vida dos mesmos. A dor física e o desconforto psicológico foram os domínios mais prevalentes.

A comparação do presente estudo com demais evidências científicas encontradas na literatura sinalizou a necessidade de mais estudos que permitam associações de marcadores clínicos e outros questionários de avaliação epidemiológica, com o impacto da saúde bucal na qualidade de vida.

As investigações que avaliam a qualidade de vida poderão culminar em mudanças nas políticas de saúde e em uma nova perspectiva para os programas educacionais em saúde.

\section{Referências}

1. Silveira MF, Marôco JP, Freire RS, Martins AMEBL, Marcopito, LF. Impacto da saúde bucal nas dimensões física e psicossocial: uma análise através da modelagem com equações estruturais. Cad. Saúde Pública, 2014 Jun; 30(6):1-15.

2. Souza LV, Abrão LSO, Novaes MSP, Loureiro RMP, Castro AM, Oliveira FS. Percepção dos pais sobre a qualidade de vida relacionada à saúde bucal de crianças com paralisia cerebral. Horizonte Científico, 2010; 4(2): 1-29.

3. Oliveira BM, Mininel VA, Felli VEA. Qualidade de vida de graduando em enfermagem. Rev Bras Enferm, 2011Jan-Fev; 64(1): 130-5.
4. Castanha AR, Coutinho MPL, Saldanha AAW, Ribeiro CG. Avaliação da qualidade de vida em soropositivos para o HIV. Estud. psicol., 2007 Jan-Mar; 24(1): 23-31.

5. Barbosa TS, Mialhe FL, Castilho ARF, Gavião MBD. Qualidade de vida e saúde bucal em crianças e adolescentes: aspectos conceituais e metodológicos. PhysisRevista de Saúde Coletiva, 2010; 20(1): 283-300.

6. Seidl EMF, Zannon CMLC. Qualidade de vida e saúde: aspectos conceituais e metodológicos. Cad. Saúde Pública, 2004 MarAbr; 20(2): 580-88.

7. Carvalho AMC, Araújo TME. Conhecimento do adolescente sobre vacina no ambiente de estratégia de saúde da família. RevBrasEnferm, 2012 Mar-Abr; 65(2): 229-35.

8. Organização Mundial de Saúde. Organização Pan-Americana da Saúde. Família e Saúde. Tema 4.7 da Agenda Provisória, 55으 Sessão do Comitê Regional/ 44ạ Conselho Diretor. Washigton, 14p., setembro, 2003.

9. Matos MS, Monteiro LS, Bomfim RT, Matos RS. Hábitos de higiene bucal e dieta de adolescentes de escolas públicas e privadas em Salvador, Bahia. Revista Brasileira de Ciências da Saúde, 2009; 13(2):7-14.

10. Tesch FC, Oliveira BH, Leão $A$. Mensuração do impacto dos problemas bucais sobre a qualidade de vida de crianças: aspectos conceituais e metodológicos. Cad. Saúde Pública, 2007; 23(11):2555-64.

11. Slade GD. Derivation and validation of a short-form oral health impact profile. Community Dent Epidemiol. 1997; 25: 284-90.

12. Barbieri $\mathrm{CH}$, Rapoport A. Avaliação da qualidade de vida dos pacientes reabilitados com próteses implanto-muco-suportadas versus próteses totais convencionais. Revista Brasileira Cirurgia Cabeça e Pescoço, 2009; 38(2): 84-7.

13. Bastos RS, Carvalho ES, Xavier A, Caldanha ML, Bastos JRM, Lauris JRP. Dental caries related to quality of life in two Brazilian adolescent groups: a cross-sectional randomised study. International Dental Journal 2012; 62: 137-143.

14. Miotto MHMB, Barcellos LA, Velten DB. Avaliação do impacto na qualidade de vida causado por problemas bucais na população adulta e idosa em município da Região Sudeste. Ciência \& Saúde Coletiva, 2012; 17(2):397-406.

15. Krisdapong $\mathrm{S}$, Prasertsom P, Rattanarangsima K, Adulyanon S, Sheiham A. Setting oral health goals that include oral health- 
related quality of life measures: a study carried out among adolescents in Thailand. Cad. Saúde Pública, 2012 Out; 28(10):1881-92.

16. Monteiro AX. Relação do medo, dor, ansiedade e condições de saúde bucal com o acesso aos serviços de saúde bucal e qualidade de vida de adolescentes. São Paulo. Tese [Doutorado] - Faculdade de Odontologia de Bauru. Universidade de São Paulo, 2013.

17. Ferreira CA, Loureiro CA, Araújo VE. Propriedades psicométricas de indicador subjetivo aplicado em crianças. Rev Saúde Pública, 2004; 38(3): 445-52

18. Feu D, Oliveira BH, Sales HX, Miguel JAM. Más-oclusões e seu impacto na qualidade de vida de adolescentes que buscam tratamento ortodôntico. Ortodontia SPO, 2008; 41(4): 35565.

19. Brasil. Ministério da Saúde. Secretaria de Atenção à Saúde. Departamento de Atenção Básica. Projeto SB Brasil 2010: pesquisa nacional de saúde bucal-resultados principais. Brasília (DF): Ministério da Saúde; 2011; 68 p.

\section{Endereço para Correspondência}

Universidade Estadual do Sudoeste da Bahia UESB

Rua Av. José Moreira Sobrinho - Jequiezinho, Jequié - BA

CEP.: 45205-490

e-mail: capcarvalho@uesb.edu.br

Recebido em 02/03/2017

Aprovado em 28/11/2017

Publicado em 06/12/2017 